\title{
Severidad del trauma, optimismo, crecimiento postraumático y bienestar en sobrevivientes de un desastre natural*
}

\author{
Severity of Trauma, Optimism, Posttraumatic Growth \\ and Well-Being in Survivors of a Natural Disaster
}

Recibido: noviembre 9 de 2012 | Revisado: abril 9 de 2013 | Aceptado: junio 9 de 2013

\author{
FElipe EduARdo García MartíneZ** \\ Alejandro ReYes ReYeS *** \\ Universidad Santo Tomas, Santiago, Chile \\ FELIX COVA SOLAR ***** \\ Universidad de Concepción, Chile
}

doi:10.11144/Javeriana.UPSY13-2.stop

Para citar este artículo: García, F. E., Reyes, A. \& Cova, F. (2014). Severidad del trauma, optimismo, crecimiento postraumático y bienestar en sobrevivientes de un desastre natural. Universitas Psychologica, 13(2). doi:10.11144/Javeriana.UPSY13-2. stop

* El proyecto fue revisado y aprobado por el Comité del Doctorado en Psicología de la Universidad de Concepción.

** Coordinador Carrera de Psicología, Universidad Santo Tomás, Concepción, Chile. Correo electrónico: felipegarciam@yahoo.es

*** Jefe de Carrera Psicología, Universidad Santo Tomás, Chile. Correo electrónico: areyesr@santotomas.cl

****** Vicedecano Facultad de Ciencias Sociales, Universidad de Concepción, Chile. Correo electrónico: fecova@udec.cl
RESUMEN

El propósito del estudio fue evaluar, en una población afectada por un desastre natural, la relación de algunas variables sociales y psicológicas con el bienestar psicológico y el crecimiento postraumático. Se obtuvo una muestra de 446 personas de la provincia de Concepción (Chile), afectadas por el terremoto del 27/F del 2010. Se emplearon el Inventario de Crecimiento Postraumático (PTGI), el Cuestionario de Orientación Vital (LOT-R) y la Flourishing Scale. Se analizó la relación entre severidad objetiva del evento (pérdida material, daño físico en alguien cercano y daño físico personal), severidad subjetiva del evento (considerar el evento como traumático o que alteró sus vidas) y optimismo disposicional, observándose que la interacción del optimismo con la severidad subjetiva u objetiva aporta significativamente en la predicción del bienestar y el crecimiento, moderando el impacto de dichas variables. Además, se evaluaron diferencias por sexo, edad y nivel socioeconómico. Se determinó la influencia relativa del nivel socioeconómico sobre el crecimiento postraumático. Este estudio resalta la importancia de las condiciones sociales sobre los efectos psicológicos de los desastres naturales y la importancia del optimismo para amortiguar dichos efectos. Palabras clave autores

Trauma psicológico, optimismo, bienestar, crecimiento postraumático, terremoto. Palabras clave descriptors

\begin{abstract}
A B S T R A C T
The aim of the study was to evaluate, in a population affected by a natural disaster, the relationship of social and psychological variables with the psychological well-being and posttraumatic growth. Participated 446 people of the province of Concepcion, Chile, affected by the earthquake on 27/F, 2010. We used the Posttraumatic Growth Inventory (PTGI), the Life Orientation Test (LOT-R) and the Flourishing Scale. We analyzed the relationship between objective severity of the event (material loss, physical damage to someone close and personal physical damage), subjective severity of the event (considering the event as traumatic or altered their lives) and dispositional optimism, showing that the interaction of optimism with subjective or objective severity contributes significantly to the prediction of well-being and growth, moderating the impact of these variables. In addition, we evaluated differences by sex, age and socioeconomic status. There was a relative influence of socioeconomic status on posttraumatic growth. This study shows the relevance of social conditions on the psychological
\end{abstract}


effects of natural disasters and the importance of optimism to mitigate these effects.

Keywords authors

Psychological trauma, optimism, well-being, posttraumatic growth, earthquake.

Keywords plus

Los desastres naturales generan un gran impacto en la vida de las personas y comunidades (Cova \& Rincón, 2010). Esto explica el interés que ha despertado el estudio de las consecuencias psicopatológicas de la exposición a este tipo de desastres, entre las que se encuentran el trastorno de estrés postraumático, la depresión y el consumo abusivo de alcohol y drogas, entre otras (García, 2011). Diversos aspectos han sido relacionados con diferencias interpersonales en la respuesta a situaciones de desastre natural. Como factores que inciden en una mayor vulnerabilidad psicopatológica en supervivientes de terremotos y tsunamis, han sido señalados: a) sexo femenino (Batniji, Van Ommeren \& Saraceno, 2005; John, Russell \& Russell, 2007; Tang, 2006; Tural et al., 2001; Xu \& Song, 2011), b) bajos ingresos o bajo nivel educativo (Xu \& Song, 2011), c) severidad objetiva del sismo: haber sufrido la pérdida de vidas o de bienes como el hogar, haber sufrido lesiones físicas, ser testigo de la muerte de alguien cercano, estar sin comida o agua, desplazamiento prolongado (Dewaraja \& Kawamura, 2006; Irmansyah, Dharmono, Maramis \& Minas, 2010; John et al., 2007; Sumer, Karanci, Berument \& Gunes, 2005; Tang, 2006; Tural et al., 2001; Wickrama \& Kaspar, 2007) y d) severidad subjetiva del sismo: riesgo percibido, miedo a morir o ser herido, falta de control percibido, evaluación negativa de la respuesta de estrés (por ejemplo, verlo como señal de debilidad personal), interpretación negativa de los recuerdos del trauma (Batniji et al., 2005; Figueroa, Marín \& González, 2010; Lommen, Sanders, Buck \& Arntz, 2009; Roussos et al., 2005; Tural et al., 2001; Sumer et al., 2005; Wahlström, Michélsen, Schulman \& Backheden, 2008; Xu \& Song, 2011).

En las últimas décadas, ha surgido un interés complementario por estudiar no solo las consecuencias psicopatológicas de los desastres, sino también su impacto sobre aspectos como el bienestar, definido como el óptimo funcionamiento psicológico y experiencial de las personas (Ryan \& Dici, 2001), y el crecimiento postraumático, referido a la posibilidad de que las personas experimenten cambios positivos como resultado de vivir estas experiencias adversas (Tedeschi \& Calhoun, 1996). Este interés radica en la posibilidad de prevenir malestar psicológico, implementar programas de intervención temprana en afectados por desastres de diversa índole y promover una mejor calidad de vida, por la asociación encontrada entre el bienestar, el crecimiento y ajuste psicológico en personas expuestas a un evento traumático (Páez et al., 2011). Existe evidencia de bienestar psicológico (Stratta et al., 2012) y de crecimiento postraumático (García, Jaramillo, Martínez, Valenzuela \& Cova, 2013) en personas expuestas a un terremoto, lo que lleva a preguntarse por las variables psicológicas relacionadas con estas consecuencias positivas en lugar de las respuestas psicopatológicas ya mencionadas.

Entre las variables que podrían incidir en el bienestar y el crecimiento, se encuentra el optimismo disposicional, un rasgo relativamente consistente y estable que lleva a expectativas favorables acerca de las cosas que le suceden a uno en la vida (Vera-Villarroel, Córdova \& Celis, 2009), y que ha aparecido en algunos estudios relacionándose negativamente con la ansiedad en población general (Pavez, Mena \& Vera-Villarroel, 2012) y con la sintomatología postraumática en sobrevivientes de desastres (Organización Panamericana de la Salud, 2006; Sumer et al., 2005; Vernberg et al., 2008). El optimismo ha mostrado tener una influencia positiva sobre el bienestar psicológico y subjetivo (Vera-Villarroel, Pavez \& Silva, 2012).

El propósito de la presente investigación fue estudiar a personas afectadas por el terremoto y tsunami de Chile del 27/F del año 2010. Para esto, se evaluaron cómo variables sociodemográficas (sexo, edad, nivel socioeconómico) y la severidad, tanto objetiva como subjetiva del trauma vivido, se relacionaban con el crecimiento postraumático y el bienestar psicológico, tras la experiencia de este desastre, así como el rol del optimismo disposicional en dicha relación. 


\section{Método}

\section{Diseño}

Se empleó un diseño correlacional. Se evaluó a los participantes en el conjunto de variables consideradas, entre 19 a 21 meses luego de ocurrido el evento.

\section{Participantes}

El total de participantes fue de 446 sujetos, 213 varones y 233 mujeres de la provincia de Concepción, Chile. El promedio de edad correspondió a 41 años (DE $=14.3$, rango 18 a 65 años). Todos los participantes estuvieron expuestos al terremoto del 27/F de 2010 y reconocen este evento como traumático para sus vidas (lo que se midió a través de dos preguntas elaboradas por los autores sobre severidad subjetiva del sismo). Fueron seleccionados a través de un muestreo por accesibilidad por cuotas, buscando su representatividad en cuanto a sexo, edad y nivel socioeconómico (Tabla 1).

\section{Instrumentos}

\section{Postraumatic Growth Inventory (PTGI)}

Este instrumento fue diseñado por Tedeschi y Calhoum (1996) para la medición del Crecimiento Postraumático. Consta de 21 ítems que se responden en una escala Likert de 0 a 5 puntos. La versión utilizada fue la traducción al español de la escala publicada por Páez et al. (2011), la que fue retraducida al inglés por un experto independiente para observar su adecuación con la versión original, a fin de estudiar las propiedades psicométricas del instrumento en población expuesta al terremoto de Chile (García, Cova \& Melipillán, en prensa). En el presente estudio se utilizan los puntajes de la escala total, la que tuvo una consistencia interna alta $(\alpha=0.95)$.

\section{Flourishing Scale (FS)}

Fue creada por Diener et al. (2009) para medir el bienestar psicológico, definido como un indicador de la satisfacción con la vida y que específicamente representa el funcionamiento humano óptimo. Se empleó la traducción al español de Melipillán y Ramírez, quienes observaron adecuadas propiedades psicométricas en población adulta chilena (comunicación personal, 2011). Está conformada por 8 ítems que se responden en una escala Likert de 1 (muy en desacuerdo) hasta 5 puntos (muy de acuerdo). En el estudio original, el FS obtuvo una alta consistencia interna $(\alpha=0.86)$. En el presente estudio, se obtuvo un $\alpha$ de 0.8 .

\section{Test de Orientación Vital (LOT-R)}

Elaborado por Scheier, Carver y Bridges (1994) para la medición del Optimismo Disposicional. Este instrumento consta de 6 ítems más 4 de relleno, en una escala Likert de 5 puntos, tres de ellos en sentido positivo (optimismo) y tres en sentido negativo (pesimismo), los que se revierten para ob-

TABLA 1

Características sociodemográficas de los participantes

\begin{tabular}{|c|c|c|c|c|c|c|c|}
\hline & $18-30$ & AÑOS & $31-50$ & AÑOS & $51-65$ & AÑOS & \multirow{2}{*}{$\begin{array}{c}\text { TOTAL POR } \\
\text { NSE } \\
\text { (Porcentaje) }\end{array}$} \\
\hline & HOMBRE & MUJER & HOMBRE & MUJER & HOMBRE & MUJER & \\
\hline NSEa BAJO & 20 & 25 & 23 & 39 & 21 & 32 & $160(36)$ \\
\hline NSE MEDIO & 45 & 27 & 35 & 33 & 19 & 35 & $194(43.5)$ \\
\hline NSE ALTO & 15 & 16 & 15 & 15 & 20 & 11 & $92(20.5)$ \\
\hline $\begin{array}{l}\text { TOTAL POR EDAD } \\
\text { (Porcentaje) }\end{array}$ & \multicolumn{2}{|c|}{$148(33)$} & \multicolumn{2}{|c|}{$160(36)$} & \multicolumn{2}{|c|}{$138(31)$} & 446 \\
\hline
\end{tabular}

${ }^{a}$ Nivel socioeconómico.

Fuente: elaboración propia. 
tener una puntuación total orientada hacia el polo de optimismo. Fue valorada como adecuada para población chilena por Vera-Villarroel et al. (2009) quienes obtuvieron una consistencia interna media $(\alpha=0.65)$. En el presente estudio su consistencia interna fue de $\alpha=0.45$.

\section{Severidad objetiva del evento}

Esta variable fue medida a través de tres preguntas elaboradas por los autores para explorar condiciones relacionadas con desastres naturales según la literatura revisada. Estas preguntas evaluaban daño en la propiedad, daño físico personal y daño físico en alguien cercano. Cada pregunta era respondida en una escala Likert de 5 puntos. El puntaje de esta variable se obtiene con la suma total de las tres preguntas, en un rango entre 0 y 12 puntos.

Severidad subjetiva del evento

Esta variable fue medida a través de dos preguntas elaboradas por los autores que medían si la persona percibía que el evento había alterado su vida y si percibía el evento como traumático. Cada pregunta era respondida en una escala Likert de 5 puntos con rango de 0 a 8 puntos. Fueron utilizadas en un estudio con estudiantes universitarios expuestos al mismo terremoto (García et al., 2013) y en pobladores expuestos a violencia policial en un conflicto social en Chile (García, Capponi, Hinrichs, Lillo, Rodríguez \& Sánchez,
2012), obteniendo un $\alpha$ de 0.69 en el primer caso y de 0.74 , en el segundo.

\section{Cuestionario sociodemográfico}

Incluyó información relativa a sexo y edad y preguntas acerca del ingreso por persona en el hogar (ingreso per cápita), a fin de determinar su nivel económico.

\section{Procedimiento}

Los participantes fueron encuestados por alumnos de psicología capacitados para tal efecto entre septiembre y noviembre del año 2011, a través del procedimiento de accesibilidad por cuotas. Previo a la aplicación de la encuesta, se solicitó a los participantes firmar un consentimiento informado en el que se aseguraba la voluntariedad, el anonimato y la confidencialidad de los datos entregados. El proyecto fue revisado y aprobado por el Comité del Doctorado en Psicología de la Universidad de Concepción.

\section{Análisis de datos}

En una primera etapa se realizó un análisis descriptivo de la población y de los instrumentos utilizados. Para probar las hipótesis de estudio, se realizaron dos regresiones lineales múltiples, una con el crecimiento postraumática y otra con el bienestar psicológico como variables dependientes. Se empleó el software PASW Statistics 18 de SPSS.

TABLA 2

Estadísticos descriptivos de las variables de estudio $(n=446)$

\begin{tabular}{l|c|c|c|c|}
\hline \multicolumn{1}{c|}{ Variables } & M & DE & Mín. & Máx. \\
\hline Bienestar psicológico & 34.12 & 4.03 & 20 & 40 \\
\hline Crecimiento postraumático & 68.72 & 22.58 & 6 & 105 \\
\hline Severidad subjetiva & 5.44 & 1.36 & 4 & 8 \\
\hline Severidad objetiva & 4.43 & 2.45 & 0 & 12 \\
\hline Optimismo disposicional & 15.13 & 3.31 & 5 & 24 \\
\hline Edad & 40.94 & 14.29 & 18 & 65 \\
\hline Ingreso per cápita & 217.414 & 266.449 & 5.250 & 3.000 .000 \\
\hline
\end{tabular}

Fuente: elaboración propia. 


\section{Resultados}

En la Tabla 2 se presentan los estadísticos descriptivos de las variables de estudio.

Las correlaciones de las variables se presentan en la Tabla 3. El optimismo disposicional, el crecimiento postraumático y el bienestar psicológico aparecen correlacionadas positivamente entre sí en forma baja o moderada, lo que da cuenta que se trata de constructos relacionados pero diferentes. Severidad objetiva y subjetiva se relacionan moderadamente entre sí $(r=0.37 p<0.001)$. El crecimiento postraumático muestra relaciones débiles pero significativas con el ingresoper cápita $(r=0.16$, $p<0.001)$, con la severidad subjetiva del evento $(r$ $=0.19, p<0.001)$ y con la severidad objetiva $(r=$ $0.11, p<0.05)$. Por su parte, el bienestar psicológico presenta relaciones significativas con el ingreso per cápita $(r=0.1, p<0.05)$ y con la severidad objetiva del evento $(r=-0.13, p<0.01)$.

Comparando a hombres y mujeres en el conjunto de variables estudiadas, se observaron diferencias significativas en severidad subjetiva del evento $t(444)=3.097, p<0.01$ y crecimiento postraumáti$\cot (444)=2.268, p<0.05$, obteniendo en ambas mayores puntajes las mujeres $(\mathrm{M}=5.63, \mathrm{DE}=$ 1.393 y $M=71.03, D E=22.661$, respectivamente) que los hombres $(M=2.24, D E=1.300$ y $M=$ 66.2, $\mathrm{DE}=22.271$, respectivamente).

Para evaluar la relación y el efecto de las variables severidad subjetiva del evento, severidad objetiva del evento, optimismo disposicional, edad, sexo e ingreso per cápita, sobre las variables bienestar psicológico y crecimiento post-traumático, se llevó a cabo un análisis de regresión lineal múltiple (RLM). Se siguió un procedimiento de pasos sucesivos, de modo que las variables no significativas fueron saliendo del modelo (Hair, Anderson, Tatham \& Black, 1999).

El modelo de regresión respecto de bienestar psicológico obtuvo un coeficiente $R^{2}$ de $0.255\left(R^{2}\right.$ adj $=0.248$ ) y una adecuada significación estadística $\left(F_{4,441}=37.771, p<0.001\right)$. El modelo indica que existe una relación lineal directa entre los predictores severidad subjetiva del evento y optimismo disposicional con los puntajes de bienestar psicológico, existiendo interacción entre severidad subjetiva y optimismo disposicional, así como entre severidad subjetiva y severidad objetiva; los valores de los coeficientes del modelo se presentan en la Tabla 4.

Al comparar el modelo en los tres niveles socioeconómicos de la muestra, se observa que para el nivel socioeconómico bajo resultan significativas las variables predictoras optimismo disposicional y severidad objetiva del evento $\left(F_{(2,157)}=21.631, p<\right.$ 0.001), conservándose solo el optimismo disposicional como variable predictora en la submuestra de nivel socioeconómico medio $\left(F_{(1,192)}=67.682 ; p<\right.$ 0.001) y en el nivel socio económico alto $\left(F_{(1,91)}=\right.$ 17.301, $p<0.001$ ).

El modelo de regresión obtenido al considerar el crecimiento postraumático como variable respuesta, muestra una relación más débil con las variables predictoras $\left(R^{2}=0.122 ; R_{\text {adj }}^{2}=0.114\right)$, pero que igualmente da cuenta de la existencia de una relación lineal significativa entre los predictores y el

TABLA 3

Correlaciones de Pearson entre las variables de estudio

\begin{tabular}{lllllll}
\hline & $\begin{array}{c}\text { Ingreso } \\
\text { per cápita }\end{array}$ & $\begin{array}{c}\text { Severidad objetiva } \\
\text { del evento }\end{array}$ & $\begin{array}{c}\text { Severidad subjetiva } \\
\text { del evento }\end{array}$ & $\begin{array}{c}\text { Optimismo } \\
\text { disposicional }\end{array}$ & $\begin{array}{c}\text { Crecimiento } \\
\text { postraumático }\end{array}$ & $\begin{array}{c}\text { Bienestar } \\
\text { psicológico }\end{array}$ \\
\hline Edad & 0.07 & $0.11^{*}$ & $0.19^{* * *}$ & -0.03 & $-0.11^{*}$ & -0.03 \\
Ingreso per cápita & & 0.01 & -0.02 & $0.09 *$ & $0.16^{* * *}$ & $0.1^{*}$ \\
Severidad objetiva del evento & & & $0.37^{* * *}$ & $-0.1^{*}$ & $0.11^{*}$ & $-0.13^{* *}$ \\
Severidad subjetiva del evento & & & & -0.06 & $0.19^{* * *}$ & -0.06 \\
Optimismo disposicional & & & & & $0.12^{* *}$ & $0.47^{* * *}$ \\
Crecimiento postraumático & & & & & & $0.34^{* * *}$ \\
\hline
\end{tabular}

$* p<0.05$ *** $p<0.01$ *** $p<0.001$.

Fuente: elaboración propia. 
TABLA 4

Coeficientes, niveles de significación y correlaciones semiparciales del modelo de RLM sobre los puntajes de bienestar psicológico

\begin{tabular}{|c|c|c|c|c|c|}
\hline & \multicolumn{2}{|c|}{$\begin{array}{l}\text { Coeficientes no } \\
\text { estandarizados }\end{array}$} & \multicolumn{2}{|c|}{ Coeficientes estandarizados } & \multirow[b]{2}{*}{$s r$} \\
\hline & B & $\mathrm{EE}$ & $\beta$ & $t$ & \\
\hline (Constante) & 30.18 & 1.61 & & $18.8 * * *$ & \\
\hline Severidad subjetiva del evento & -1.52 & 0.58 & -0.51 & $-2.61 * *$ & -0.11 \\
\hline Optimismo disposicional & 0.27 & 0.1 & 0.22 & $2.59^{* *}$ & 0.11 \\
\hline Sev.Subjetiva x Sev. Objetiva & -0.05 & 0.02 & -0.15 & $-2.25 *$ & -0.09 \\
\hline Sev. Subjetiva x Optimismo disposicional & 0.17 & 0.04 & 0.64 & $3.28 * * *$ & 0.14 \\
\hline
\end{tabular}

$* p<0.05$. ** $p<0.01$. *** $p<0.001$.

Fuente: elaboración propia.

crecimiento postraumático $\left(F_{(4,441)}=15.342, p<\right.$ 0.001). Como se muestra en la Tabla 5 , se advierte una relación lineal directa con los predictores severidad subjetiva del evento e ingreso per cápita y una relación inversamente proporcional con la variable ingreso per cápita, existiendo interacción entre la variable severidad subjetiva del evento y optimismo disposicional, así como entre severidad objetiva del evento e ingreso per cápita.

Al estratificar la muestra por nivel socioeconómico, se encuentra que para el nivel bajo resultan significativas las variables predictoras optimismo disposicional y severidad subjetiva del evento $\left(F_{(2,159)}=16.607 ; p<0.001\right)$, conservándose solo la severidad objetiva del evento como variable predictora en la submuestra de nivel socioeconómico alto $\left(F_{(1,90)}=9.843 ; p<0.002\right)$, no siendo posible construir un modelo para el nivel socioeconómico medio.

\section{Discusión}

La relación del optimismo con el bienestar psicológico ha sido ampliamente observada en las investigaciones (Seligman, 2002), sin embargo, las explicaciones de los procesos que dan cuenta de esta relación han generado escaso consenso (VeraVillarroel et al., 2012).

En el presente estudio, se observó que la severidad subjetiva referida a un desastre natural se relaciona negativamente con el bienestar. Sin embargo, esta relación se invierte si se la hace interactuar con el optimismo. En tal sentido, el optimismo podría estar jugando un rol moderador que disminuye el impacto de las condiciones de vulnerabilidad sobre la satisfacción con la vida de los afectados.

También se observa un efecto de interacción entre la severidad objetiva y subjetiva del sismo, potenciándose entre ellas. Al parecer, la severidad

\section{TABLA 5}

Coeficientes, niveles de significación y correlaciones semiparciales del modelo de RLM sobre los puntajes de crecimiento postraumático

\begin{tabular}{|c|c|c|c|c|c|}
\hline & \multicolumn{2}{|c|}{$\begin{array}{l}\text { Coeficientes no } \\
\text { estandarizados }\end{array}$} & \multicolumn{2}{|c|}{ Coeficientes estandarizados } & \multirow[b]{2}{*}{$s r$} \\
\hline & B & $\mathrm{EE}$ & $\beta$ & $t$ & \\
\hline (Constante) & 67.16 & 2.37 & & $28.32 * * *$ & \\
\hline Ingreso per cápita & 0 & 0 & -0.47 & $-5.26 * * *$ & -0.23 \\
\hline Severidad subjetiva del evento & -0.46 & 1.74 & -0.27 & $-2.62 * *$ & -0.12 \\
\hline Sev. Subjetiva*Optimismo disposicional & 0.46 & 0.11 & 0.46 & $4.39 * * *$ & 0.2 \\
\hline Sev. Objetiva*Ingreso per cápita & 0 & 0 & 0.35 & $3.85 * * *$ & 0.17 \\
\hline
\end{tabular}

$* p<0.05$. ** $p<0.01$. *** $p<0.001$.

Fuente: elaboración propia. 
objetiva del sismo influye en el bienestar en la medida que hay interacción con la severidad subjetiva. Al estratificar el análisis por nivel socioeconómico, se observa que la severidad objetiva solo tiene influencia en los niveles bajos, lo que indica que para los otros niveles; las pérdidas materiales no parecen impactar negativamente en su bienestar. Esto confirma lo planteado por Sanhueza, Contreras y Denis (2012) quienes señalan que los desastres naturales tienen mayor impacto sobre el bienestar de personas pobres, debido a la ausencia de seguros que permitan amortiguar dichos impactos.

Respecto al crecimiento postraumático, por tratarse de un constructo relativamente reciente, su relación con el optimismo ha sido menos explorada, aunque Ho et al. (2011) y Bozo, Gundogdu y Buyukasik-Colak (2009) encontraron una relación positiva en pacientes con distintos tipos de cáncer. En este estudio, se confirma la relación positiva y se establece además que, al igual que en el caso del bienestar, la severidad subjetiva se relaciona negativamente con el crecimiento, pero al interactuar con el optimismo, dicha relación se invierte. El optimismo también podría estar jugando un rol moderador que permite que quienes consideren el desastre natural como un evento traumático y que alteró sus vidas, son capaces también de percibir cambios positivos a partir de dicha experiencia extrema.

La influencia del nivel socioeconómico sobre el crecimiento postraumático se observa negativa, es decir, a mayores ingresos menor crecimiento. La evidencia disponible no ha encontrado que exista una relación significativa entre ingresos y crecimiento en población general (Helgeson, Reynolds \& Tomich, 2006) ni en pacientes con cáncer (Windows, Jacobsen, Booth-Jones \& Fields, 2005). Es probable que en población afectada por un desastre natural mayores ingresos impliquen menos cambios, producto de ese evento. Sin embargo, al hacerla interactuar con la severidad objetiva, esta relación se invierte y se hace positiva, indicando que si las personas de mayores ingresos experimentan pérdidas significativas pueden percibir, paradojalmente, más cambios positivos en su vida a partir de dicha experiencia.
Una limitación de este estudio es la baja fiabilidad de la escala para medir optimismo. La baja consistencia interna obtenida está en concordancia con lo observado en otras investigaciones (Anadón, 2006; Augusto, Aguilar \& Salgedo, 2008; Grimaldo, 2004; Mera \& Ortiz, 2012), y podría explicarse por la baja cantidad de ítems que la componen (Aiken, 2003; Anastasi \& Urbina, 1998). Por su parte, Grimaldo estima que el constructo evaluado por el LOT-R posee un nivel bajo de especificidad; es decir, es más bien general y global, de tal modo que la consistencia en la medida de un constructo de esta naturaleza tiende a no ser alto, tal como señalan Bagozzi y Edwards (1998) y Bagozzi y Heatherton (1994). De todos modos, en el presente estudio se decidió continuar con los análisis utilizando este instrumento, debido a que se trata de la escala que se está utilizando en Chile y Latinoamérica para la medición del optimismo, lo que permite la comparación de resultados, pero a su vez nos plantea el desafío de hacer una revisión exhaustiva del constructo y posibilitar la elaboración de una escala distinta, más fiable y robusta. Como lo señala Cohen y Swerdlik (2006), si la fiabilidad de una prueba es relativamente baja, puede ser poco práctico incrementar el número de reactivos para alcanzar un nivel de fiabilidad aceptable, siendo una mejor alternativa elaborar una escala adecuada que permita comparar estos resultados y eventualmente confirmarlos.

Una segunda limitación radica en el diseño transversal del estudio, lo que impide esclarecer la direccionalidad de las relaciones, y un tercer aspecto que se debe considerar es la baja cantidad de variables evaluadas, que no permiten construir un modelo predictivo de bienestar psicológico ni de crecimiento postraumático, aunque sí establecer relaciones importantes. Estudios posteriores debiesen incorporar otras variables que se ha observado se relacionan con los efectos del trauma psicológico (Calhoun, Cann $\&$ Tedeschi, 2011): como la ruptura de creencias básicas, la rumiación, el compartir socialmente la experiencia con otros, el apoyo social, diversas estrategias de afrontamiento y rasgos como la esperanza y la resistencia al estrés, entre otras, lo que contribuiría a una mejor comprensión de los mecanismos que actúan en el bienestar y el crecimiento. 


\section{Referencias}

Aiken, L. (2003). Test psicológicos y evaluación. México: Pearson.

Anastasi, A. \& Urbina, S. (1998). Test psicológicos. México: Prentice Hall.

Anadón, O. (2006). Inteligencia emocional percibida y optimismo disposicional en estudiantes universitarios. Revista Electrónica Interuniversitaria de Formación del Profesorado, 9(1), 1-13.

Augusto, J., Aguilar, M. \& Salguero, M. (2008). The role of perceived emotional intelligence and dispositional optimism/pessimism in social problem solving: A study of social work students. Electronic Journal of Research in Educational Psychology, 6(2), $363-382$

Bagozzi, R. \& Edwards, J. (1998). A general approach for representing constructs in organizational research. Organizational Research Methods, 1(1), 45-87.

Bagozzi, R. \& Heatherton, T. (1994). A general approach to representing multifaceted personality constructs: Application to state self-esteem. Structural Equation Modelling, 1(1), 35-67.

Batniji, R., Van Ommeren, M. \& Saraceno, B. (2006). Mental and social health in disasters: Relating qualitative social science research and the sphere standard. Social Science \&O Medicine, 62(8), 1853. 1864.

Bozo, O., Gundogdu, E. \& Buyukasik-Colak, C. (2009). The moderating role of different sources of perceived social support on the dispositional optimism - posttraumatic growth relationship in postoperative breast cancer patients. Journal of Health Psychology, 14, 1009-1020.

Calhoun, L. G., Cann, A. \& Tedeschi, R. G. (2011). The posttraumatic growth model: Sociocultural considerations. En T. Weiss \& R. Berger (Eds.), Posttraumatic growth and culturally competent practice (pp. 1-14). New York: Wiley \& Sons.

Cohen, R. \& Swerdlik, M. (2006). Pruebas y evaluación psicológicas. Introducción a las pruebas y a la medición (6a. ed.). México: McGraw Hill.

Cova, F. \& Rincón, P. (2010). El terremoto y tsunami del 27-F y sus efectos en la salud mental. Terapia Psicológica, 28(2), 179-185.
Dewaraja, R. \& Kawamura, N. (2006). Trauma intensity and posttraumatic stress: Implications of the tsunami experience in Sri Lanka for the management of future disasters. International Congress Series, 1287, 69-73.

Diener, E., Wirtz, D., Tov, W., Kim-Prieto, C., Choi, D., Oishi, S. \& Biswas-Diener, R. (2009). New measures of well-being: Flourishing and positive and negative feelings. Social Indicators Research, 39, 247-266.

Figueroa, R., Marín, H. \& González, M. (2010). Apoyo psicológico en desastres: propuesta de un modelo de atención basado en revisiones sistemáticas y metaanálisis. Revista Médica de Chile, 138(2), 143-151.

García, F. (2011). Prevention of psychopathological consequences in survivors of tsunamis. En M. Mokhtari (Ed.), Tsunami: A growing disaster (pp. 211-232). Rijeka, Croatia: InTech.

García, F., Capponi, C., Hinrichs, F., Lillo, F., Rodríguez, C. \& Sánchez, J. (2012, noviembre). Violencia policial y resistencia. Afrontamiento y crecimiento postraumático en habitantes de Aysén. Ponencia presentada en el VII Congreso Chileno de Psicología, Valparaíso, Chile.

García, F., Cova, F. \& Melipillán, R. (en prensa). Propiedades psicométricas del Inventario de Crecimiento Postraumático en población chilena afectada por un desastre natural. Revista Mexicana de Psicología.

García, F., Jaramillo, C., Martínez, A. M., Valenzuela, I. \& Cova, F. (2013, julio). Modelo estructural explicativo de los procesos psicológicos que conducen al estrés y al crecimiento postraumático. Póster aceptado en el XXXIV Congreso de la Sociedad Interamericana de Psicología, Brasilia, Brasil.

Grimaldo, M. (2004). Niveles de optimismo en un grupo de estudiantes de una universidad particular de la ciudad de Lima. Liberabit, 10(10), 97-107.

Hair, J. F., Anderson, R. E., Tatham, R. L. \& Black, W. C. (1999). Análisis multivariante (5a. ed.). Madrid: Prentice Hall.

Helgeson, V., Reynolds, K. \& Tomich, P. (2006). A meta-analytic review of benefit finding and growth. Journal of Consulting and Clinical Psychology, 74(5), $797-816$. 
Ho S. Rajandram, R. K., Chan, N., Samman, N., McGrath, C. \& Zwahlen, R. (2011). The roles of hope and optimism on posttraumatic growth in oral cavity cancer patients. Oral Oncology, 47(2), 121-124.

Irmansyah, I., Dharmono, S., Maramis, A. \& Minas, H. (2010). Determinants of psychological morbidity in survivors of the earthquake and tsunami in Aceh and Nias. International Journal of Mental Health Systems, 4(1), 4-8.

John, P., Russell, S. \& Russell, P. (2007). The prevalence of posttraumatic stress disorder among children and adolescents affected by tsunami disaster in Tamil Nadu. Disaster Management $\mathcal{E}$ Response, 5(1), 3-7.

Lommen, M., Sanders, A., Buck, N. \& Arntz, A. (2009). Psychosocial predictors of chronic Post-Traumatic Stress Disorder in Sri Lankan tsunami survivors. Behaviour Research and Therapy, 47, 60-65.

Mera, P. \& Ortiz, M. (2012). La relación del optimismo y las estrategias de afrontamiento con la calidad de vida de mujeres con cáncer de mama. Terapia Psicológica, 30(3), 69-78.

Organización Panamericana de la Salud. (2006). Guía Práctica de salud mental en situaciones de desastre. Washington, DC: Autor.

Páez, D., Vázquez, C., Bosco, S., Gasparre, A., Iraurgi, I. \& Sezibera, V. (2011). Crecimiento post-estrés y post-trauma: posibles aspectos positivos y beneficiosos de la respuesta a los hechos traumáticos. En D. Páez, C. Martín Beristain, J. L. González, N. Basabe \& J. de Rivera (Eds.), Superando la violencia colectiva y construyendo cultura de paz (pp. 311-339). Madrid: Fundamentos.

Pavez, P., Mena, L. \& Vera-Villarroel, P. (2012). El rol de la felicidad y el optimismo como factor protector de la ansiedad. Universitas Psychologica, 11(2), 369-380.

Roussos, A., Goenjian, A., Steinberg, A., Sotiropoulou, C., Kakaki, M., Kabakos, C., Karagianni, S., et al. (2005). Posttraumatic stress and depressive reactions among children and adolescents after the 1999 earthquake in Ano Liosia, Greece. American Journal of Psychiatry, 162(3), 530-537.

Ryan, R. \& Deci, E. (2001). On happiness and human potentials: A review of research on hedonic and eudaimonic well-being. Annual Review of Psychology, 52, 141-166.

Sanhueza, C., Contreras, D. \& Denis, A. (2012). Terremoto y sus efectos sobre el bienestar: un análisis multidimensional. Persona y Sociedad, 26(1), 43-66.

Scheier, M., Carver, C. \& Bridges, M. (1994). Distinguishing optimism from neuroticism (and trait anxiety, self-mastery, and self-esteem): A re-evaluation of the Life Orientation Test. Journal of Personality and Social Psychology, 67(6), 1063-1078.

Seligman, M. (2002). La auténtica felicidad. Barcelona: Ediciones B.

Sumer, N., Karanci, N., Berument, S. \& Gunes, H. (2005). Personal resources, coping self-efficacy, and quake exposure as predictors of psychological distress following the 1999 earthquake in Turkey. Journal of Traumatic Stress, 18(4), 331-342.

Tang, C. (2006). Positive and negative postdisaster psychological adjustment among adult survivors of the Southeast Asian earthquake-tsunami. Journal of Psychosomatic Research, 61(5), 699-705.

Tedeschi, R. G. \& Calhoun, L. G. (1996). The posttraumatic growth inventory: Measuring the positive legacy of trauma. Journal of Traumatic Stress, 9(3), 455-471.

Tural, U., Aybar Tolun, H., Karakaya, I., Erol, Y., Yildiz, M. \& Erdoğan, S. (2001). Predictors of current comorbid psychiatric disorders with posttraumatic stress disorder in earthquake survivors. Turkish Journal of Psychiatry, 12, 175-183.

Vera-Villarroel, P., Córdova, N. \& Celis, K. (2009). Evaluación del optimismo: un análisis preliminar del Life Orientation Test versión revisada (LOTR) en población chilena. Universitas Psychologica, 8(1), 61-68.

Vera-Villarroel, P., Pavez, P. \& Silva, J. (2012). El rol predisponente del optimismo: hacia un modelo etiológico del bienestar. Terapia Psicológica, 30 (2), 77-84.

Vernberg, E. M., Steinberg, A. M., Jacobs, A. K., Brymer, M. J., Watson, P. J., Osofsky, J. D., Layne, C., et al. (2008). Innovations in disaster mental health: Psychological first aid. Professional Psychology: Research and Practice, 39(4), 381-388.

Wahlström, L., Michélsen, H., Schulman, A. \& Backheden, M. (2008). Different types of exposure to 
the 2004 tsunami are associated with different levels of psychological distress and posttraumatic stress. Journal of Traumatic Stress, 21(5), 463-470.

Wickrama, K. \& Kaspar, V. (2007). Family context of mental health risk in Tsunami-exposed adolescents: Findings from a pilot study in Sri Lanka. Social Science EO Medicine, 64(3), 713-723.
Windows, M., Jacobsen, P., Booth-Jones, M. \& Fields, K. (2005). Predictors of posttraumatic growth following bone marrow transplantation for cancer. Health Psychology, 24(3), 266-273.

Xu, J. \& Song, X. (2011). Posttraumatic stress disorder among survivors of the Wenchuan earthquake 1 year after: Prevalence and risk factors. Comprehensive Psychiatry, 52(4), 431-437. 